\title{
Perfil de Salud de los Trabajadores de una Planta Procesadora de Olefinas del Estado Zulia, Venezuela
}

\author{
A profile of workers' health in an olefins plant in the \\ Venezuelan state of Zulia
}

\author{
Ricardo Portillo, Monika Bellorín, Yadira Sirit y Carlos Acero
}

Instituto de Medicina del Trabajo e Higiene Industrial de la Universidad del Zulia, Maracaibo, Venezuela. monikabellorin@cantv.net

Recibido 19 Junio 2007/Enviado para Modificación 29 Diciembre 2007/Aceptado 23 Enero 2008

\section{RESUMEN}

Objetivo Determinar el perfil de salud de los trabajadores de una Planta procesadora de Olefinas del Estado Zulia.

Métodos Se realizó un estudio observacional descriptivo, evaluando a 142 trabajadores de las áreas administrativa- técnica, operaciones y mantenimiento. A cada trabajador se le realizó una historia médico ocupacional, examen físico, exámenes de laboratorio, audiometría y evaluación visual. El grado de obesidad se determinó mediante la clasificación de la OMS, el riesgo cardiovascular se estableció aplicando las tablas de riesgo de las Sociedades Europeas.

Resultados El promedio de edad de los trabajadores fue de 36,2 $\pm 6,9$ años. Los individuos del área de operaciones mostraron promedio de peso, y tensión arterial sistólica más elevada que el resto de las áreas. Se determinó una alta frecuencia de factores de riesgo cardiovascular predominando el consumo de alcohol $(89,4$ $\%)$, obesidad (62,7\%), hipertrigliceridemia (46,5\%), y presión arterial sistólica elevada (45,1\%); siendo los operadores los más afectados. Se encontró una frecuencia moderada de trabajadores con enzimas hepáticas alteradas AST (38 \%) y GGT $(29,6 \%)$. Las patologías más frecuentes fueron ametropia $(64,1 \%)$, dislipidemia (57,8 \%), obesidad I (50 \%), e hipertensión (45,1 \%). Los mayores de 40 años mostraron cifras de tensión arterial sistólica más elevadas. Se encontró una correlación positiva y significativa entre IMC y tensión sistólica.

Conclusión Existe una alta prevalencia de factores de riesgo cardiovascular. Se recomienda diseñar y aplicar acciones de promoción de la salud, encaminadas a modificar los estilos de vida para disminuir el riesgo de enfermedad cardiovascular.

Palabras Clave: Perfil de salud, trabajadores, Venezuela (fuente: DeCS, BIREME).

ABSTRACT

Objective Determining the profile of health for people working in an olefin plant.

Methods This was an observational, descriptive study carried out on 142 workers from technical-administrative, operator and maintenance areas. Each worker's 
employment and medical history was examined; they had a physical examination and laboratory, audiometric and visual tests. The degree of obesity was determined by WHO classification; cardiovascular risk was classified by using the European Societies' risk table.

Results The workers had a mean age of $36,2 \pm 6,9$. The operators presented mean weight and systolic pressure which was higher than that of the rest of the workers. A high risk factor frequency was determined for cardiovascular pathologies: alcohol consumption $(89,4 \%)$, obesity $(62,7 \%)$, hypertriglycerides in the blood $(46,5 \%)$ and high systolic pressure (45,1\%); operators were the most affected workers. A moderate frequency of workers having altered hepatic enzymes, AST (38\%) and GGT $(29,6 \%)$ were observed The most frequently encountered pathologies were ametropia (64,1\%), dyslipidaemia (57,8 \%), obesity (50\%) and hypertension $(45,1 \%)$. Workers aged more than 40 presented higher systolic pressure. A significant correlation between IMC and systolic pressure was found.

Conclusion A high prevalence of cardiovascular risk factors was found. Healthcare should be designed and promoted and action should be taken towards modifying lifestyles, aimed at reducing the risk of cardiovascular disease.

Key Words: Health, worker, Venezuela (source: MeSH, NLM).

$\mathrm{E}$ l hombre en la relación de sus funciones laborales, está relacionado a condiciones generales y especiales bajo las cuales realiza su trabajo, incluyendo aspectos organizativos y funcionales de las empresas y empleadores, métodos, sistemas y procedimientos, así como servicios sociales, y factores externos al medio ambiente laboral que tienen influencia sobre él, determinando las condiciones del trabajo y del ecosistema relacionado con su ocupación (1).

El ser humano es una unidad biológica, psicológica y social, influenciado por su herencia y el medio ambiente que lo rodea, por tanto la salud como la enfermedad dependen del equilibrio armónico entre el hombre, factores de riesgo, el ambiente laboral y el comunitario. La salud, es un estado de completo bienestar físico, mental y social y no sólo la ausencia de enfermedades, en el que el individuo es capaz de desarrollar sus potencialidades creativas. Sin embargo numerosas personas tienen una visión más limitada y no ven en la salud otra cosa que la ausencia de enfermedad $(2,3)$.

La industrialización y el trabajo han tenido consecuencias negativas para la salud de los trabajadores. Esos efectos se derivan directamente de la exposición a factores de riesgo, los cuales pueden ser físicos, químicos, biológicos y psicosociales, entre otros. Los factores de riesgo, aunados a los diferentes procesos en las industrias y a las características sociales e individuales del 
trabajador, condicionan el perfil de salud de la población laboral. Entre las razones que justifican el patrocinio por parte de las empresas de los programas de promoción de la salud de los trabajadores figuran el bienestar de los individuos, prevención de pérdida de productividad debido a enfermedades o incapacidades y al absentismo asociado y el control de los costos de los seguros de enfermedad (4)

La planta procesadora de Olefinas de la Industria Petroquímica de Venezuela, es de importancia en nuestro país ya que ella genera la materia prima para la industria del plástico a nivel nacional e internacional. Esta planta cuenta con una mano de obra joven en el área productiva, sometida a la exposición a agentes físicos, químicos, biológicos, mecánicos, psicosociales y disergonómicos potencialmente peligrosos para la salud del trabajador; éstos asociados a las exigencias del trabajo y a los factores biológicos y de tipo comportamental propios del individuo, generan un perfil salud-enfermedad que es necesario conocer y abordar.

Debido a lo anteriormente expuesto, el propósito de este estudio fue determinar el perfil de salud de los trabajadores de la planta procesadora de Olefinas, que permita precisar su atención e intervención inmediata por parte del personal médico, así como, incentivar a los trabajadores a participar activamente en un programa de promoción y fomento de la salud, de prevención de enfermedades y reducción de los factores de riesgo cardiovascular.

\section{MÉTODOS}

Se realizó un estudio descriptivo en los trabajadores de una planta de Olefinas de la industria Petroquímica de Venezuela del Estado Zulia, entre Julio a Diciembre de 2005.

Se invitaron a participar a todos los trabajadores masculinos de la planta con antigüedad laboral mayor o igual a 1 año $(n=156)$, de los cuales 142 (91 \%) aceptaron voluntariamente a participar en el estudio y firmaron un consentimiento luego de ser informados de los objetivos de la investigación. Los trabajadores fueron clasificados en tres categorías: Administrativo - técnico (Coordinadores, supervisores, ingenieros de proceso, ingenieros de producción, planificadores y panelistas), Operaciones (operadores de compresores, operadores de refrigeración, operadores de fraccionamiento, operadores de hornos y calderas, operadores de efluentes, almacén y servicio) y mantenimiento (mecánicos, instrumentistas y electricistas). 
A cada participante se le realizó una historia médico ocupacional estructurada en cinco partes: a) datos del trabajador que incluyen las variables sociodemográficas, puesto de trabajo, y antigüedad laboral, b) antecedentes ocupacionales, personales y familiares, c) examen físico completo, d) resultados de exámenes de laboratorio y e) resultados de estudios complementarios.

En el examen físico se determinó la tensión arterial utilizando un tensiómetro de columna de mercurio, la misma fue medida en el brazo derecho en tres oportunidades luego que el trabajador guardara reposo sentado por cinco minutos y un intervalo entre las mediciones igual o mayor a dos minutos, asumiendo como valor de la misma, el promedio de las tres mediciones; se consideró hipertenso a todos aquellos sujetos con tensión arterial sistólica mayor o igual a 140 mm Hg, tensión arterial diastólica mayor o igual a 90 mmHg, (5) o con antecedente de diagnóstico médico para hipertensión arterial.

Las definiciones de obesidad y sobrepeso fueron hechas siguiendo los criterios de la OMS, considerándose sobrepeso cuando el índice de masa corporal (IMC) es de 25 a 29.9, Obesidad I (IMC de 30 a 34.9), Obesidad II (IMC de 35 a 39.9) y Obesidad III (IMC $\geq 40$ ) (6).

El riesgo coronario a los diez años, fue calculado aplicando las tablas de riesgo de las sociedades europeas (7); estas diferenciadas por sexo utilizan la edad, colesterol total, tabaquismo, tensión arterial y diabetes mellitus como factores de riesgo cardiovascular. El nivel de riesgo viene representado en una tabla en colores, considerando por consenso un riesgo alto si alcanza o supera el $20 \%$ a los 10 años.

Así mismo, a cada trabajador se le tomó una muestra de 15 cc de sangre venosa periférica con ayuno previo de 10 a 12 horas, para determinar por métodos enzimáticos comerciales de Human GMBH, Alemania, los niveles de triglicéridos (GPO-PAP Method), colesterol total (CHOD-PAP Method), glicemia (GOD-PAP Method) y pruebas de función hepática: Alanino amino transferasa (ALT), (Aspartato amino transferasa (AST), Gamma glutamil transpeptidasa (GGT) y bilirrubina (Ciba-corning Dianostic). Además se les realizó a todos los sujetos hematología completa. Se consideraron como valores normales aquellos menor que $200 \mathrm{mg} / \mathrm{dl}$ de colesterol total y menor que 150 $\mathrm{mg} / \mathrm{dl}$ de triglicéridos; glicemia en ayunas menor o igual a $110 \mathrm{mg} / \mathrm{dl}$. Con respecto a las pruebas de función hepática, se utilizaron los siguientes valores de referencia: TGP/ALT 0-30 UI/ml, TGO/AST 0-30 UI/ml, GGT 7-30 UI/ml, bilirrubina total $0.3-1.0 \mathrm{mg} / \mathrm{ml}$ y bilirrubina conjugada $0.3 \mathrm{mg} / \mathrm{ml}$. 
En relación a los estudios complementarios, se les practicó audiometría, la cual se realizó en cabina hermética capaz de atenuar el ruido ambiental, utilizándose un audiómetro modelo Beltone 112, para medir vía aérea y vía ósea, para lo cual el trabajador se encontraba por lo menos 24 horas anterior al estudio sin exposición al ruido y sin sintomatología de resfriado común, otitis, amigdalitis o cualquier otra sintomatología de vías respiratorias superiores.

El examen visual se llevó a cabo utilizando un aparato de evaluación modelo screnocope, Topcon SS-3, capaz de medir visión cercana, lejana y foria, se valoró que el trabajador al momento del examen no presentara procesos infecciosos oculares.

Se utilizó estadística descriptiva (números absolutos, porcentajes, medidas de tendencia central y de dispersión), se aplicaron pruebas de diferencias de media Z, Chi cuadrado, y coeficiente de correlación de Pearson a los resultados obtenidos, considerándose estadísticamente significativos los resultados con una p $<0,05$. Para el procesamiento de los datos se empleó el paquete estadístico SPSS versión 10 para Windows.

\section{RESULTADOS}

De la población de 156 trabajadores masculinos de la Planta de Olefinas, invitados a participar en el estudio, un total de 142 (91 \%) fueron evaluados. El promedio de edad fue de 36,2 \pm 6,9 años, en un rango desde 20 a 54 años, y una antigüedad en la empresa de 10,9 \pm 6,9 años con rango desde 12 meses a 32 años.

En la Tabla 1 se indican los valores promedios de las características antropométricas y sociodemográficas por área de trabajo. El grupo de trabajadores del área de operaciones mostró promedios más elevados de todas las variables exceptuando la talla. Sin embargo al aplicar ANOVA se obtuvo solo diferencia significativa para el peso, entre los trabajadores de acuerdo a las áreas de trabajo $(\mathrm{p}<0,05)$.

Los factores de riesgo cardiovascular más frecuentes detectados al examen físico y pruebas de laboratorio fueron el consumo de alcohol (89,4 \%), la obesidad (62,7\%), valores elevados de triglicéridos (46,5\%), y cifras tensionales sistólicas elevadas (45,1\%). Es importante resaltar que los operadores poseen la mayor proporción de factores de riesgo. 
Los factores de riesgo cardiovascular, distribuidos por grupos de edad se muestran en la Tabla 2. Se observa que los mayores de 40 años representan el estrato con mayor número de trabajadores y muestran una mayor prevalencia tanto de presión arterial sistólica $(\mathrm{n}=24)$, como diastólica elevada $(\mathrm{n}=7)$, así mismo, colesterol total $>200 \mathrm{mg} / \mathrm{dl}(\mathrm{n}=19)$, glicemia por encima de $110 \mathrm{mg} / \mathrm{dl}$ ( $\mathrm{n}=8$ ), y triglicéridos mayor de $150 \mathrm{mg} / \mathrm{dl}(\mathrm{n}=25)$. Es importante destacar que los trabajadores que mayormente obtuvieron IMC por encima de 30, se encuentran en los estratos de mayores de 40 años y 31 y 35 años. Al aplicar ANOVA, se obtuvo solo diferencia significativa para las variables presión arterial diastólica $(\mathrm{p}<0,05)$.

Tabla 1. Características antropométricas y sociodemográficas por áreas de una planta procesadora de Olefinas del Estado Zulia, 2005

\begin{tabular}{lccc}
\hline \multicolumn{1}{c}{ Variable } & $\begin{array}{c}\text { Mantenimiento } \\
(\mathrm{N}=34) \\
\mathrm{X} \pm \mathrm{DE}\end{array}$ & $\begin{array}{c}\text { Operaciones } \\
(\mathrm{N}=71)\end{array}$ & $\begin{array}{c}\text { Técnico } \\
(\mathrm{N}=37)\end{array}$ \\
\hline Edad & $36,1 \pm 7,4$ & $36,5 \pm 6,3$ & $35,7 \pm 7,6$ \\
Antigüedad laboral & $9,5 \pm 7,6$ & $12,0 \pm 6,0$ & $10,0 \pm 7,7$ \\
Peso* & $87,0 \pm 14,1$ & $92,0 \pm 12,0$ & $84,2 \pm 14,6$ \\
Talla & $1,7 \pm 0,1$ & $1,7 \pm 0,1$ & $1,7 \pm 0,1$ \\
IMC & $30,0 \pm 4,1$ & $31,5 \pm 3,4$ & $29,7 \pm 4,4$ \\
\hline X Promedio; DE = Desviación estándar, IMC= Índice de masa corporal; * $\mathrm{p}<0,05$
\end{tabular}

Tabla 2. Factores de riesgo cardiovascular distribuidos por grupos de edad, en trabajadores de una planta procesadora de Olefinas del estado Zulia, 2005

\begin{tabular}{|c|c|c|c|c|c|c|c|c|c|c|c|c|}
\hline \multirow[b]{3}{*}{ Factor de riesgo } & \multicolumn{12}{|c|}{ Grupo de edad (años) } \\
\hline & \multicolumn{2}{|c|}{$<25$} & \multicolumn{2}{|c|}{$26-30$} & \multicolumn{2}{|c|}{$31-35$} & \multicolumn{2}{|c|}{$36-40$} & \multicolumn{2}{|c|}{$>40$} & \multicolumn{2}{|c|}{ TOTAL } \\
\hline & $\mathrm{n}$ & $\%$ & $\mathrm{n}$ & $\%$ & $n$ & $\%$ & $n$ & $\%$ & $\mathrm{n}$ & $\%$ & $n$ & $\%$ \\
\hline Alcohol & 4 & 2,8 & 24 & 16,9 & 34 & 23,9 & 28 & 19,7 & 37 & 26,1 & 127 & 89,4 \\
\hline$I M C>30$ & 1 & 0,7 & 17 & 12,0 & 25 & 17,6 & 18 & 12,7 & 28 & 19,7 & 89 & 62,7 \\
\hline Triglicéridos > 150 & - & - & 11 & 7,7 & 16 & 11,3 & 14 & 9,9 & 25 & 17,6 & 66 & 46,5 \\
\hline PAS $>130$ & $=$ & $=$ & 7 & 4,9 & 21 & 14,8 & 12 & 8,5 & 24 & 16,9 & 64 & 45,1 \\
\hline Colesterol $>200$ & 1 & 0,7 & 4 & 2,8 & 17 & 12,0 & 9 & 6,3 & 19 & 13,4 & 50 & 35,2 \\
\hline $\mathrm{PAD}>85^{\circ}$ & $=$ & $=$ & 1 & 0,7 & 6 & 4,2 & 5 & 3,5 & 7 & 4,9 & 19 & 13,4 \\
\hline Tabaquismo & 1 & 0,7 & 3 & 2,1 & 4 & 2,8 & 4 & 2,8 & 6 & 4,2 & 18 & 12,7 \\
\hline Glicemia > 110 & - & $=$ & $=$ & $=$ & 1 & 0,7 & 3 & 2,1 & 8 & 5,6 & 12 & 8,5 \\
\hline
\end{tabular}

PAS= Presión arterial sistólica; PAD= Presión arterial Diastólica; *p<0,05

Los promedios de los parámetros antropométricos, de pruebas de laboratorio y tensión arterial de los trabajadores por grupo de edad se presentan en la Tabla 3. Los trabajadores mayores de 40 años presentan valores más elevados de tensión arterial sistólica y diastólica, volumen corpuscular medio, glicemia, colesterol, triglicéridos, y bilirrubina total. Los trabajadores con edades comprendidas entre 31 y 35 años, mostraron valores más elevados de peso, IMC, ALT, AST, y GGT. Los trabajadores entre 36 y 40 años mostraron valores 
más elevados de cuenta blanca y plaquetas. Y los trabajadores con edades comprendida entre 26 y 30 años mostraron valores más elevados de hemoglobina. Sin embargo, al aplicar ANOVA se obtiene diferencia significativa solo para las variables, peso, y tensión arterial sistólica $(\mathrm{p}<0,05)$.

Al evaluar las pruebas complementarias, se observó que el 64,1 \% de los trabajadores presentaron la prueba visual alterada y el 32,4 \% mostró alteración en los resultados de la prueba audiométrica. Se determinó que los operadores presentaron un porcentaje mayor de pruebas visuales (33,8\%) y audiométricas alteradas (15,5\%), sin embargo al aplicar Chi cuadradado no se obtuvo diferencia significativa entre los trabajadores por áreas de trabajo ( $\mathrm{p}>0,05)$.

Tabla 3. Parámetros antropométricos, de laboratorio y presión arterial de los trabajadores por grupo de edad de una planta procesadora de

\begin{tabular}{lccccc}
\multicolumn{5}{c}{ Olefinas del Estado Zulia, 2005} \\
\hline & $<25$ & $26-30$ & $31-35$ & $36-40$ & $>40$ \\
VARIABLE & $X \pm D E$ & $X \pm D E$ & $X \pm D E$ & $X \pm D E$ & $X \pm D E$ \\
\hline PESO* & $74,4 \pm 23,0$ & $85,6 \pm 12,2$ & $91,6 \pm 11,1$ & $90,6 \pm 13,2$ & $89,0 \pm 14,5$ \\
TALLA & $1,7 \pm 0,1$ & $1,7 \pm 0,1$ & $1,7 \pm 0,0$ & $1,7 \pm 0,1$ & $1,7 \pm 0,1$ \\
IMC & $27,0 \pm 3,9$ & $29,4 \pm 3,7$ & $31,8 \pm 3,6$ & $31,0 \pm 3,6$ & $30,8 \pm 4,1$ \\
PAS* & $110,0 \pm 7,1$ & $119,3 \pm 10,8$ & $127,3 \pm 12,6$ & $126,1 \pm 13,8$ & $127,7 \pm 16,8$ \\
PAD & $68,0 \pm 4,5$ & $74,3 \pm 7,7$ & $79,5 \pm 8,5$ & $80,6 \pm 10,9$ & $81,8 \pm 10,2$ \\
Hb & $12,9 \pm 0,9$ & $13,4 \pm 0,8$ & $13,4 \pm 1,0$ & $13,3 \pm 0,8$ & $12,9 \pm 1,1$ \\
VCM & $82,5 \pm 3,6$ & $84,8 \pm 3,9$ & $86,0 \pm 3,6$ & $85,4 \pm 4,9$ & $86,4 \pm 4,1$ \\
CB & $6,0 \pm 1,3$ & $6,6 \pm 1,2$ & $7,1 \pm 2,2$ & $7,4 \pm 2,0$ & $7,0 \pm 1,8$ \\
GLIC & $83,4 \pm 5,3$ & $87,9 \pm 8,7$ & $91,0 \pm 8,9$ & $104,0 \pm 53,0$ & $104,1 \pm 39,2$ \\
Colesterol & $157,0 \pm 31,0$ & $169,2 \pm 24,0$ & $189,9 \pm 38,4$ & $191,7 \pm 34,1$ & $194,4 \pm 37,2$ \\
Trigliceridos & $70,4 \pm 36,6$ & $129,5 \pm 78,7$ & $163,4 \pm 104,1$ & $218,0 \pm 156,2$ & $212,8 \pm 119,2$ \\
ALT & $20,4 \pm 13,9$ & $22,5 \pm 10,3$ & $27,1 \pm 13,2$ & $24,1 \pm 11,1$ & $25,5 \pm 15,3$ \\
AST & $35,6 \pm 47,5$ & $31,9 \pm 19,9$ & $54,1 \pm 42,8$ & $38,1 \pm 19,3$ & $38,1 \pm 26,6$ \\
FAlcalina & $60,0 \pm 12,1$ & $67,8 \pm 18,3$ & $68,9 \pm 15,5$ & $76,1 \pm 23,0$ & $71,3 \pm 21,7$ \\
BT & $0,4 \pm 0,1$ & $0,7 \pm 0,4$ & $0,7 \pm 0,2$ & $0,7 \pm 0,3$ & $0,9 \pm 0,7$ \\
BD & $0,1 \pm 0,0$ & $0,1 \pm 0,0$ & $0,1 \pm 0,0$ & $0,1 \pm 0,1$ & $0,1 \pm 0,1$ \\
& $261000 \pm$ & $254233,3 \pm$ & $264675,7 \pm$ & $284000 \pm$ & $261769,2 \pm$ \\
PLAQ & 53329,2 & 36501 & 45560,7 & 56490,7 & 50224,5 \\
\hline
\end{tabular}

$X=$ Promedio; $\mathrm{DE}=$ Desviación estándar; $\mathrm{PAS}=$ Presión arterial sistólica; $\mathrm{PAD}=$ Presión arterial diastólica; $\mathrm{Hb}=$ Hemoglobina; $\mathrm{VCM}=$ Volumen corpuscular medio; $\mathrm{CB}=$ cuenta blanca; $\mathrm{ALT}=$ Alanino amino transferasa; $\mathrm{AST}=$ Aspartato amino transferasa; $\mathrm{GGT}=$ Gamma glutamil transpeptidasa; $\mathrm{BT}=$ Bilirrubina total; $\mathrm{BD}=$ Bilirrubina directa; * $p<0,05$

En relación a los diagnósticos más frecuentemente determinados, la ametropia ocupa el primer lugar (64,1\%), seguido de dislipidemia (57,8 \%), obesidad I (50,0 \%) e hipertensión arterial (45,1 \%). Destaca que si se agrupan los trabajadores con diagnostico de obesidad I, II y III, esta patología alcanza el segundo lugar de prevalencia con 62,7\%; los operadores presentaron la mayor frecuencia de los mencionados diagnósticos. 
Con respecto al riesgo coronario en 10 años, se determinó que todos los individuos con 29 años o menos ( $\mathrm{n}=25)$, tuvieron riesgo bajo. En el grupo de 30 a 39 años ( $n=68), 67$ presentaron riesgo bajo y 1 riesgo ligero, mientras que en los trabajadores con cuarenta años y más ( $\mathrm{n}=49$ ), se observó que 39 tuvieron riesgo bajo, 1 riesgo ligero y 9 riesgo moderado.

Se relacionaron las variables cuantitativas, a través del coeficiente de correlación de Pearson, y se obtuvo una correlación positiva entre edad y las variables peso $(\mathrm{r}=0,2)$, tensión sistólica $(\mathrm{r}=0,2)$, tensión diastólica $(\mathrm{r}=0,3)$, glicemia $(\mathrm{r}=0,2)$, colesterol $(\mathrm{r}=0,3)$ y triglicéridos $(\mathrm{r}=0,3)$. Así mismo se obtuvo correlación positiva entre peso con las variables tensión sistólica $(\mathrm{r}=0,4)$, ALT $(r=0,2)$, AST $(r=0,3)$, y GGT $(r=0,2)$. De igual manera se encontró correlación positiva entre IMC con tensión sistólica $(\mathrm{r}=0,4),(\mathrm{p}<0,05)$.

\section{DISCUSIÓN}

Desde el punto de vista ocupacional, es innegable la importancia que tiene el hecho de conocer los riesgos a los que se exponen los trabajadores para diseñar los programas de prevención de riesgos laborales en las empresas, para ello es necesario definir un patrón inicial del estado de salud de los trabajadores que sirva como punto de referencia para estudios posteriores, y para poder precisar el momento en el que un individuo se aleja de las características que se consideran normales para el grupo y permita actuar de manera oportuna, evitando o minimizando las complicaciones o daños irreparables. Por otro lado, la determinación del perfil de salud brinda criterios que pueden orientar en el desarrollo de condiciones ideales de trabajo (8).

Los resultados de este estudio muestran una población de trabajadores masculinos, adultos jóvenes, en su mayoría menores de 40 años, con una antigüedad en el área de trabajo en promedio de mas o menos 10 años, lo cual brinda un buen periodo de exposición a los factores de riesgo de la planta de Olefinas.

Al comparar las variables antropométricas y sociodemográficas de los trabajadores de las diferentes áreas, se encontró que los del área de operaciones obtuvieron los valores más elevados en todas las variables con respecto al resto de las áreas operativas de la planta, sin embargo, únicamente existió diferencia significativa entre los grupos en cuanto a la variable peso. Hecho que podría ser explicado por el horario de labor, donde el personal operativo labora por turnos rotativos, lo cual conlleva a dificultad para fijar un horario 
para el desarrollo de actividades deportivas, adicionalmente la actividad de campo les origina agotamiento físico, que no favorece la realización de ejercicios posterior a la jornada de trabajo, además el trabajador por turno tiende a consumir alimentos con elevado contenido de carbohidratos y grasas, lo cual se traduce en aumento de la ingesta calórica, repercutiendo directamente sobre la ganancia de peso.

Los operadores mostraron valor promedio de IMC de 31,5, y los mantenedores de 30,0 siendo clasificados como obesos, mientras que los del área técnico-administrativa presentaron cifra promedio de 29,7, clasificándolos como sobrepeso, observándose una elevada frecuencia de obesidad en los sujetos evaluados. Actualmente la prevalencia de obesidad va en ascenso en la mayoría de países y es considerada como una epidemia global, con graves consecuencias para la salud pública del mundo, (9) ya que es un factor de riesgo para el desarrollo ulterior de enfermedades crónicas en el adulto mayor, tales como hipertensión arterial, enfermedad coronaria y diabetes tipo 2, entre otras (9-12). Así mismo, el estudio Framingham ha documentado que la obesidad es un factor de riesgo independiente para enfermedad cardiovascular (13).

Estos resultados son similares a los de Luna (14), quien observó una prevalencia elevada de obesidad e hipertensión en el personal del área operativa de un hospital en Guanajuato México. Igualmente, Nascimiento (15) encontró una alta prevalencia de obesidad e hipertensión arterial en trabajadores de un centro de enseñanza de salud. Sin embargo, contrastan con los reportados por Parkes (16) en trabajadores costa afuera de una industria petrolera, donde se obtuvo una media del IMC de 25,6; igualmente Lighy (17) en trabajadores petroleros del Reino Unido reportaron valor promedio del IMC de 24,8.

En la presente investigación se pudo detectar la ocurrencia de varios factores de riesgo cardiovascular, entre los que destacan en orden de frecuencia el consumo de alcohol, IMC mayor de 30, hipertrigliceridemia, y presión arterial sistólica elevada; siendo el grupo de trabajadores del área operativa los más afectados, colocándolos en una situación de mayor vulnerabilidad de padecer patología cardiovascular, entre las que destaca la cardiopatía isquémica (18). Resultados similares fueron encontrados por González (19) quien observó el consumo de alcohol, presión arterial sistólica elevada, obesidad y triglicéridos elevados entre los principales riesgos cardiovasculares en los trabajadores estudiados, siendo más frecuentes en operadores. 
Un estudio realizado en Costa Rica por Hernández (20), mostró que un 76 \% de la población tuvieron dos o más factores de riesgo para enfermedad cardiovascular.

El hecho de que los trabajadores del área operativa posean mayor número de factores de riesgo cardiovascular, puede ser debido a que con la modalidad de trabajo a turnos el organismo humano modifica sus ritmos biológicos (ritmos circadianos), forzando a la persona a invertir su ciclo normal de descanso, obligándolo a ajustar sus funciones al período de actividad nocturna, acarreando cambios en las secreciones endocrinas, digestivas, en la tensión arterial y frecuencia cardiaca, entre otras. Y esto se evidencia en estudios científicos donde se demuestra que los trabajadores por turnos padecen hasta un $40 \%$ más riesgo de sufrir enfermedades cardiovasculares, trastornos cardiovasculares entre éstos la Hipertensión Arterial (21).

Es importante destacar que en esta investigación, a pesar que el colesterol se presentó elevado en el 35\% de la población, no se encontró correlación entre este parámetro y el peso; contrario a lo obtenido por Rosas, quien además de conseguir alta prevalencia de obesidad y colesterol elevado en los trabajadores de una empresa de Lima, también encontró correlación entre las variables (22).

Al evaluar los hábitos, se obtuvo que los resultados son contradictorios a los reportados por Bustos y colaboradores (23) quienes observaron que el tabaquismo era más frecuente que el hábito alcohólico en los trabajadores. Sin embargo, es similar en cuanto a la alta prevalencia de obesidad en adultos jóvenes. También Ibarra encuentra un moderado porcentaje de trabajadores con los hábitos tabáquico, ingesta de alcohol y vida sedentaria (24).

En relación a las pruebas complementarias la mayoría de los trabajadores de las diferentes áreas mostraron resultados alterados en la prueba visual, y pruebas audiométricas normales. Los trabajadores del área de operaciones presentaron mayor porcentaje de pruebas audiométricas alteradas, probablemente porque deben permanecer más tiempo en el área de producción, donde se encuentran expuestos a diversos procesos que originan ruido. Sin embargo, no se encontró diferencias significativas entre las áreas de trabajo. Estos resultados se comparan a los obtenidos por González, quien obtuvo en trabajadores del Instituto Mexicano de los Seguros Sociales, similares proporciones (19). 
Los diagnósticos más frecuentes en los trabajadores de la planta de Olefinas fueron: ametropia, dislipidemia, obesidad e hipertensión arterial, estos últimos se relacionan al alto riesgo de enfermedad cardiovascular. Así mismo se asocia a un mayor porcentaje de patologías comunes relacionadas al trabajo, encontrándose este resultado similar a los obtenidos por Palacios en trabajadores del Servicio de Transporte en México (25).

En relación a los factores de riesgo cardiovascular analizados por grupos de edad, se determinó una alta prevalencia de sobrepeso y obesidad con diferencias significativas en el grupo de 31 a 35 años y de cifras de tensión sistólica elevada en el grupo mayor de 40 años.

El coeficiente de Pearson permitió establecer correlaciones positivas significativas entre la edad y las variables tensión diastólica, colesterol y triglicéridos. Esto podría ser explicado por el hecho que a mayor edad las alteraciones metabólicas se hacen más frecuentes $(26,27)$.

El presente estudio permitió demostrar que existe una alta prevalencia de factores de riesgo cardiovascular en los trabajadores evaluados y que los diagnósticos clínicos más frecuentes fueron ametropia, dislipidemia, obesidad, e hipertensión arterial. Se recomienda diseñar y aplicar programas de educación y de promoción de la salud, así como acciones encaminadas a modificar los estilos de vida de los trabajadores para disminuir el riesgo de enfermedad cardiovascular

Agradecimientos. Al personal que labora en el servicio médico de la empresa y a los trabajadores por su participación y apoyo al desarrollo de esta investigación *

\section{REFERENCIAS}

1. Escalona E, Younnusg M, González R, Chatignt C, Seifert A. La Ergonomía como herramienta para Trabajadores y Trabajadoras. Primera edición. Venezuela: Editorial Tatunc; Universidad de Carabobo; 2002.

2. OMS Salud para todos: Protocolo de Salud para América Latina, Geneva. (Technical series No 1200); 2000.

3. Restrepo H, Málaga H. Promoción de la Salud: Como construir vida saludable, Conceptos y Definiciones, Madrid, España: Editorial Médica Panamericana; 2001.p. 24.

4. Sanz-Gallén P, Izquierdo J, Prat A. Manual de Salud Laboral. Barcelona: Editorial Springer-Verlag Ibérica, S.A.; 1995. 
5. Chobanian A. Séptimo Informe del Comité Nacional Conjunto en Prevención, Detección Evaluación y Tratamiento de la Hipertensión Arterial. Hipertensión 2003; 42: 1206-1252

6. WHO Report Obesity: Preventing and managing the Global Epidemic. WHO Consultation on Obesity. Technical series No 894. Geneva, Switzerland:WHO; 2000.

7. Wood D, De Backer G, Faergeman O, Graham I, Mancia G Pyörala K. Task Force Report. Prevention of coronary heart disease in clinical practice: Recomendations of the second joint task force of the joint European Societies on coronary prevention. Eur Heart J 1998; 19: 1434-1503.

8. Rodríguez J. Perfil de salud y condiciones de trabajo en personal masculino de enfermería en un servicio ambulante de atención de emergencias. Noviembre 2000 [Internet] Disponible en: http://www.monografias.com/trabajos16/perfil-salud/perfilsalud2.shtml. Consultado 30-04-05

9. James PT. Obesity: Rhe wordwide epidemia. Clin Dermatol 2004; 22: 276-280.

10. Must A, Spadano J, Coakley E, Field E, Colditz G, Diezt W. The disease burden associated with overweigt and obesity. JAMA 1999; 282: 1523-1529

11. World Health Organization. Physical Status: The use and interpretation of anthropometry: Report of a WHO Expert Committee. WHO Technical Report Series No 854, Geneva, Switzerland: WHO; 1995.

12. Maggio CS, Pi-Sunyer X. The prevention and treatment of obesity. Diabetes Care 1997; 20:1744-1771.

13. Hubert HB, Feinleib M, McNamara PM, Castelli WP. Obesity as an independent risk factor for cardiovascular disease: a 26 year follow-up of participants in the Framingham Herat Study. Circulation 1983; 67: 968-977.

14. Luna M. Evaluación del estado de nutrición de los trabajadores de la unidad de medicina familiar no. 47 de León, Guanajuato. [Internet] Disponible en: http:// www.salud de los trabajadores. Consultado 12-08-05.

15. Nascimento l, Mendes I. Perfil de salud de los trabajadores de un centro de educación de salud. Rev. Lat Amer de Enfermagem 2002; 10 (4): 502-508.

16. Parkes K. Demographic and lifestyle predictors of body mass index among offshore oil industry workers: cross-sectional and longitudinal findings. Occup Med 2003; 53:213-221.

17. Lighy IM, Gibson M. Percentage body fat and prevalence of obesity in a UK offshore population. Brithish J Nutrition 1986; 56: 97-104.

18. Peñalver E, Dueñas A, Dieste W, Nordet P. Influencia de los factores de riesgo coronario en la incidencia de cardiopatía isquémica. Rev Cubana Med Gen Integr 1999; 15(4):368-371.

19. González A. Características de salud física de trabajadores de una empresa de autotransporte. [Internet] Disponible: http://www.salud de los trabajadores. Consultado 12-08-05. 
20. Hernández WI. Factores de riesgo de enfermedad cardiovascular en una población obrera industrial de la provincia de Cartago. Rev Costarric Salud Pública 2000; 9:55-64.

21. Eguía A, Baldera C, González M. Turno de noche y salud: análisis para determinar los posibles factores de riesgo asociados al bajo desempeño físico. Rev Lat Salud en el Trabajo 2001; 1(1): 37-44.

22. Rosas-Aguirre A, Lama G. Prevalencia de obesidad e hipercolesterolemia en trabajadores de una empresa de servicios de luz. Rev Peru Med Exp Salud Publica 2002; 19(Supl): 87-95.

23. Bustos P, Amigo H, Arteaga A, Acosta AM, Rona R. Factores de riesgo de enfermedad cardiovascular en adultos jóvenes. Rev Chile 2003; 131:973-980.

24. Ibarra G. Género y la salud del docente: una visión alternativa. [Internet] Rev Educ y Cult 2004. Sección 47 del SNTE. Jalisco. Mexico. Disponible en: http:// www.latarea.com.mx/articu/articu8/ibarra6.htm. Consultado: 10-08-05.

25. Palacios A. Condiciones de trabajo y salud de los trabajadores del STC Metro. [Internet] Disponible en: http://www.salud de los trabajadores. Consultado 12-12-04.

26. Foz M, Barbany M, Remesar X, Carrillo M, Aranceta J, García-Luna P, et al. Consenso SEEDO'2000, para la Evaluación del Sobrepeso y la Obesidad y el Establecimiento de Criterios de Intervención Terapéutica. . Med Clin 2000; 115: 587-597.

27. Álvarez CA. Las tablas de riesgo cardiovascular. Una revisión crítica. Med Fam 2001; 11(3): 122-139. 